\title{
Free vibration analysis for a pipe conveying fluid with intermediate support and carrying multiple concentrated masses
}

\author{
M. Z. Salim*, T. A. El-Sayed, E. M. Rabeih and S. H. Farghaly
}

Department of Mechanical Design, Faculty of Engineering, Mataria, Helwan University,

*Corresponding author. E-mail addresses: magdyzain512@yahoo.com (M. Z. Salim).

\begin{abstract}
In the present paper, the exact vibration characteristics of multi-span pipe conveying fluid has been studied. A mathematical model and a computer program are used to obtain the results. Results of typical cases have been obtained using the current model and compared with the relevant published results. The effect of intermediate support location on the vibration characteristics of clamped-pinnedpinned pipe carrying concentrated masses is evaluated. Natural frequencies and the critical flow velocity of the selected pipe are determined for different locations of both the intermediate support and the concentrated masses. Nodal and anti-nodal points of the mode shape are investigated due to the change of the intermediate support location for accepted observability and controllability aspects.
\end{abstract}

Keywords. Free vibration, pipe conveying fluid, intermediate support, concentrated masses, Bernoulli-Euler pipe and critical velocity.

\section{Introduction}

Pipes conveying fluid are main components in several industrial fields, including hydraulics, water transporting, petroleum and biological engineering, nuclear industry, fuel feeding lines in aerospace and even daily life applications. The transverse free vibration of a pipe-mass system has been investigated to show the 
effect of the concentrated masses and intermediate support location on pipe dynamic behaviors.

Ashley and Haviland [1] investigated the early substantial contribution concerned with a simply supported (pinned-pinned) span pipe. The mode shape was approximately represented by a polynomial made to satisfy the equation of motion and the system boundary conditions. Housner [2], derived the governing equation of motion for a fluid-conveying pipe. Blevins [6], provided an introduction to the analytical tools used to study the vibrations of structures exposed to a fluid flow. He also presented models used to analyze vortex-induced vibrations, oscillating flow vibrations and turbulence vibrations. Farghaly et al. [9], presented a fully coupled fluid-structure interaction model. They carried out the dynamic analysis of the piping structure using finite element analysis. They constructed the coupled equation of motion of the piping system by using the continuity and pressure constraints at each pressure discontinuity and velocity. El-Sayed et al. [10] investigated the exact natural frequencies and their associated modal shapes for an axially loaded Timoshenko stepped multi-span shaft or beam system. Farghaly et al. [11], investigated the changes in the magnitude of natural frequencies and their associated modal shapes of Timoshenko beam with respect to different system design parameters. Kang [12], studied the effects of rotary inertia of concentrated masses on the natural vibrations of a fluid-conveying pipe using theoretical modeling and numerical calculations for a clamped-supported pipe. Païdoussis [13], dealt with the dynamics of cylindrical or quasi-cylindrical, bodies in contact with axial flow-such that the structure either contains the flow or is immersed in it, or both. Kesimli et al. [14] investigated linear vibrations of a pipe carrying fluid. They study a clamped-clamped pipe with intermediated-support located in the middle section. Chellapilla et al. [15], studied free vibrations of fluid-conveying pipes resting on Pasternak-Winkler model. They investigated: pinned-pinned, clamped-pinned and clamped-clamped pipes boundary conditions. From a practical point of view, different components of a piping system, can be modelled with a simple mathematical model elements. For example there are a fluid velocity sensor, pressure sensor, temperature sensor and valves to control flow rate or any joining elements. These elements can be represented by a concentrated masses. Also, the suspension element as an elastic elements. From this point of view. The proposed mathematical model is implemented to get the following targets, (a) to study the effect of support's location and the concentrated masses carried by the pipe on the natural frequencies and associated mode shapes and (b) to estimate the critical fluid velocities which affect the stability of the system at different operating parameters. 


\section{Methodology \\ 2.1 Model assumptions}

The present model is developed considering the following assumptions [3, 4 and 8], as: (1) all displacement have been considered small so that only linear terms are considered. (2) the pipe is straight, horizontal and the flow steady, (3) the velocity profile through the constant cross-section of the pipe is neglected, (4) the fluid velocity and pressure are constants, (5) the effect of gravity on the flow through the pipe is neglected. (6) the pipe is modeled as a thin beam, the shear deformation and rotary inertia are neglected, (Bernoulli-Euler beam), (7) the material, frictional and viscous damping are not considered due to the linear limit and (8) the mass density are uniform and the pipe is inextensible.

The pipe can be modeled as a beam carrying concentrated masses with elastically intermediate support, $[7,12]$. In the present model, the pipe is modelled as a multispan beam carrying four concentrated masses which represent the pipe attached devices such as; valves, flow meters,... etc. Each span is supported elastically by a linear translational and rotational springs. The present mathematical model shown in Fig. 1a, is reduced to a case study as illustrated in Fig. (1b).

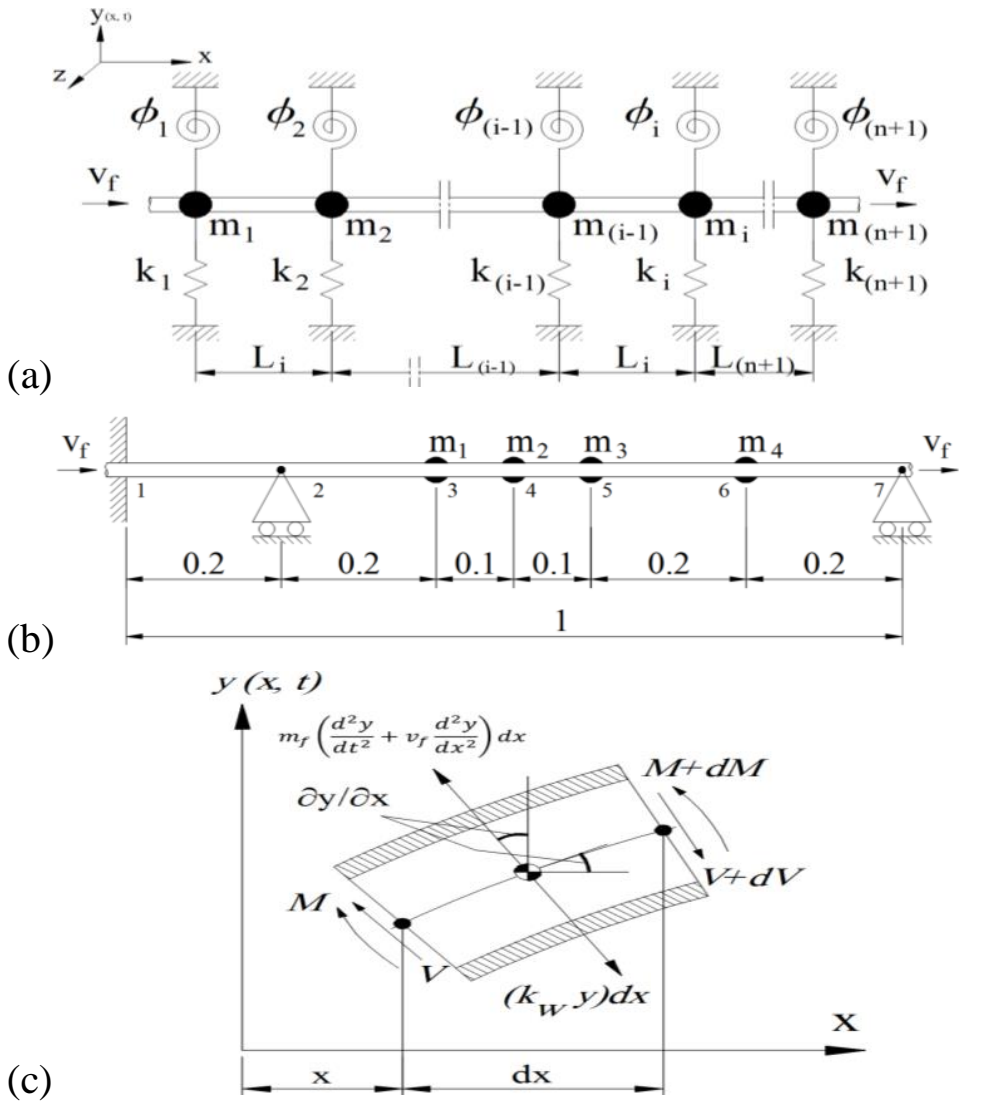

Fig. 1. Present model for a pipe conveying fluid with concentrated masses with intermediate support $[7,12]$, (a) generalized mathematical model (b) the reduced model and (c) the directions and signs of section slope, bending moment and shear force. 
The model is reduced using the following values of linear springs, rotational springs and concentrated masses. For point (1), $k_{1}=v l, \phi_{1}=v l$ and $m_{1}=v s$. For points (2\& 7), $k_{2,7}=v l, \phi_{2,7}=v s$ and $m_{2,7}=v s$. For points $(3,4,5 \& 6), k_{i}=\phi_{i}=v s$ and $m_{i}=0.1-0.4$, where $i=3,4,5$ and 6 .

\subsection{Equation of motion and solution}

Applied mathematical model for a pipe conveying fluid with length $L$ and fluid velocity $v_{f}$ is illustrated in Fig. 1 . The equation of motion for an Euler-Bernoulli pipe conveying fluid, [12 and 13] can be written as follows

$E I \frac{\partial^{4} y(x, t)}{\partial x^{4}}+\left(m_{f} v_{f}^{2}\right) \frac{\partial^{2} y(x, t)}{\partial x^{2}}+2 m_{f} v_{f} \frac{\partial^{2} y(x, t)}{\partial x \partial t}+\left(m_{p}+m_{f}\right) \frac{\partial^{2} y(x, t)}{\partial t^{2}}=0$

Where: $E=$ Young's modulus, $I=$ second moment of inertia, $y=$ lateral displacement, $m_{f}=$ fluid mass per unit length, $m_{p}=$ pipe mass per unit length. Equation (1) contains four terms defined as follows:

$E I \frac{\partial^{4} y(x, t)}{\partial x^{4}}=$ flexural restoring force, $\left(m_{f} v_{f}^{2}\right) \frac{\partial^{2} y(x, t)}{\partial x^{2}}=$ compressive axial load due to fluid motion, $2 m_{f} v_{f} \frac{\partial^{2} y(x, t)}{\partial x \partial t}=$ Coriolis term and $\left(m_{p}+m_{f}\right) \frac{\partial^{2} y(x, t)}{\partial t^{2}}=$ inertia term due to mass of fluid, pipe and concentrated mass together.

In order to simplify the problem, the Coriolis term is neglected [8]. The effect of this simplification is investigated by comparing the results with another relevant publication as illustrated in Table (2).

The non-dimensional harmonic solution for this equation is

$y_{i}(x, t)=Y_{i}(x) e^{i \omega t}$

After variables separation, the equation of motion governing each pipe span can be written in terms of mode shape displacement $Y_{i}(x)$ by substituting eq. (2) into eq. (1)

$E I Y_{i}^{\prime \prime \prime \prime}(x)+\left(m_{f} v_{f}^{2}\right) Y_{i}^{\prime \prime}(x)-\left(\left(m_{p}+m_{f}\right) \omega^{2}\right) Y_{i}(x)=0$

Eq. (3) can be written in non-dimensional form as;

$\frac{E I}{L_{i}^{4}} Y_{i}^{\prime \prime \prime \prime}(\xi)+\frac{\left(m_{f} v_{f}^{2}\right)}{L_{i}^{2}} Y_{i}^{\prime \prime}(\xi)-\left(\left(m_{p}+m_{f}\right) \omega^{2}\right) Y_{i}(\xi)=0$

$Y_{i}^{\prime \prime \prime \prime}(\xi)+\bar{v}_{f}^{2} Y_{i}^{\prime \prime}(\xi)+\lambda^{4} Y_{i}(\xi)=$

Where $\xi=x / L, \bar{v}_{f}^{2}=\left(m_{f} v_{f}^{2} L^{2}\right) / E I$ and $\lambda^{4}=\left(\left(m_{p}+m_{f}\right) \omega^{2} L^{4}\right) / E I$.

The solution of the $4^{\text {th }}$, order differential equation (5) is; 
$Y=\sum_{j=1}^{4} C_{j} e^{i \kappa_{j} \xi}$

Where $C_{j}=$ are the imaginary term constants, $\kappa_{j}=$ the wave numbers.

Substitute Eq. (6) into Eq. (5), we get

$\kappa_{j}^{4}+\bar{v}_{f}^{2} \kappa_{j}^{2}+\lambda_{i}^{4}=0$

to solve Eq. (7), applying $\kappa_{1,2}^{2}=-\frac{b}{2} \pm \sqrt{\left(\frac{b^{2}-4 a c}{2 a}\right)}$

The coefficients $a=1, b=\bar{v}_{f}^{2}$ and $c=\lambda_{i}^{4}$, then substituting into Eq. (8), the roots are

$\kappa_{1,2}=-\frac{\bar{v}_{f}^{2}}{2}+\left(\frac{\left(\bar{v}_{f}^{2}\right)^{2}}{2}-2 \lambda_{i}^{4}\right)^{1 / 2}, \quad \kappa_{3,4}=-\frac{\bar{v}_{f}^{2}}{2}-\left(\frac{\left(\bar{v}_{f}^{2}\right)^{2}}{2}-2 \lambda_{i}^{4}\right)^{1 / 2}$

The roots $\kappa_{j}$ of Eq. (7), can be presented as a function of the frequency parameter $\lambda_{i}$ and fluid velocity $\bar{v}_{f}$ substituting into Eq. $(9 \mathrm{a}, \mathrm{b})$, the solution of the ordinary differential equation (5) becomes;

$Y_{i}(\xi)=A_{1} e^{i \kappa_{1} \xi}+A_{2} e^{i \kappa_{2} \xi}+A_{3} e^{i \kappa_{3} \xi}+A_{4} e^{i \kappa_{4} \xi}$

The end conditions can be written for pipe start, intermediate and end locations as follows:

Start point 1;

Bending moment balance becomes

$$
\begin{aligned}
& (E I)_{1} \frac{\partial y_{1}^{2}(x, t)}{\partial x^{2}}-\phi_{1} \frac{\partial y_{1}(x, t)}{\partial x}=0 \\
& \left((E I)_{1} / L_{1}^{2}\right) Y_{1}^{\prime \prime}(0)-\left(\phi_{1} / L_{1}\right) Y_{1}^{\prime}(0)=0 \quad \text { or } \quad Y_{1}^{\prime \prime}(0)-\Phi_{1} Y_{1}^{\prime}(0)=0
\end{aligned}
$$

Shear force balance becomes

$$
\begin{aligned}
& (E I)_{1} \frac{\partial y_{1}^{3}(x, t)}{\partial x^{3}}-m_{i} \omega^{2} y_{1}(x, t)-k_{1} y_{1}(x, t)=0 \\
& \left((E I)_{1} / L_{1}^{3}\right) Y_{1}^{\prime \prime \prime}(0)-m_{1} L_{1} \omega^{2} Y_{1}(0)-k_{1} Y_{1}(0)=0 \\
& \text { or } \quad Y_{1}^{\prime \prime \prime}(0)-\left(m_{1}^{*} \times \lambda_{1}^{4}+Z_{1}\right) Y_{1}(0)=0
\end{aligned}
$$

Where $\Phi_{1}=\left(\phi_{1} L / E I\right), Z_{1}=\frac{k_{1} L^{3}}{E I}, \lambda_{1}^{4}=\frac{\left(m_{1} L_{1}^{4} \omega^{2}\right)}{(E I)_{1}}, m_{1}^{*}=m_{1} / L\left(m_{p}+m_{f}\right)$.

Intermediate condition point;

Displacement continuity

$$
Y_{i}(1)-Y_{(i+1)}(0)=0
$$


Slope continuity $\quad L_{(i+1) i} Y_{i}^{\prime}(1)-Y_{(i+1)}^{\prime}(0)=0$

Where $L_{(i+1) i}=L_{(i+1)} / L_{i}$

Bending moment balance: $\quad W_{1 i} \times\left(Y_{i}^{\prime \prime}(1)+\Phi_{(i+1)} Y_{i}^{\prime}(1)\right)-Y_{(i+1)}^{\prime \prime}(0)=0$

Where $W_{1 i}=(E I)_{i(i+1)} \times L_{(i+1) i}^{2},(E I)_{i(i+1)}=\frac{(E I)_{(i+1)}}{(E I)_{i}}$ and $L_{(i+1) i}^{2}=L_{(i+1)}^{2} / L_{i}^{2}$

Shear force balance $W_{2 i} \times\left(Y_{i}^{\prime \prime \prime}(1)-\left(m_{(i+1)}^{*} \times \lambda_{i}^{4}+Z_{(i+1)}\right) Y_{i}(1)\right)-Y_{(i+1)}^{\prime \prime \prime}(0)=0$ Where $W_{2 i}=\left((E I)_{i(i+1)} \times L_{(i+1) i}^{3}\right), \lambda_{i}^{4}=\left(\rho \mathrm{AA} L_{i}^{4} \omega^{2} / E_{i} I_{i}\right)$ and $L_{(i+1) i}^{3}=L_{(i+1)}^{3} / L_{i}^{3}$

End location $(\mathrm{n}+1)$

Bending moment balance: $\quad Y_{n}^{\prime \prime}(1)+\Phi_{(n+1)} Y_{n}^{\prime}(1)=0$

Shear force balance: $\quad Y_{n}^{\prime \prime \prime}(1)-\left(m_{n+1}^{*} \times \lambda_{n}^{4}+Z_{n+1}\right) Y_{n}(1)=0$

The values of spring stiffness determines the support type such as; the clamped support, $Z$ and $\Phi$ tends to infinity. The pinned support, $Z$ tends to infinity, $\Phi$ tends to zero and for the free support, $Z$ and $\Phi$ tends to zero. For harmonic solution and from the above equations, a generalized matrix $(4 \mathrm{SNx} 4 \mathrm{SN})$ for multi-span pipe can be obtained. The obtained matrix, can represents the characteristic equation, from which we can get the natural frequencies of the system, figure 2 . 


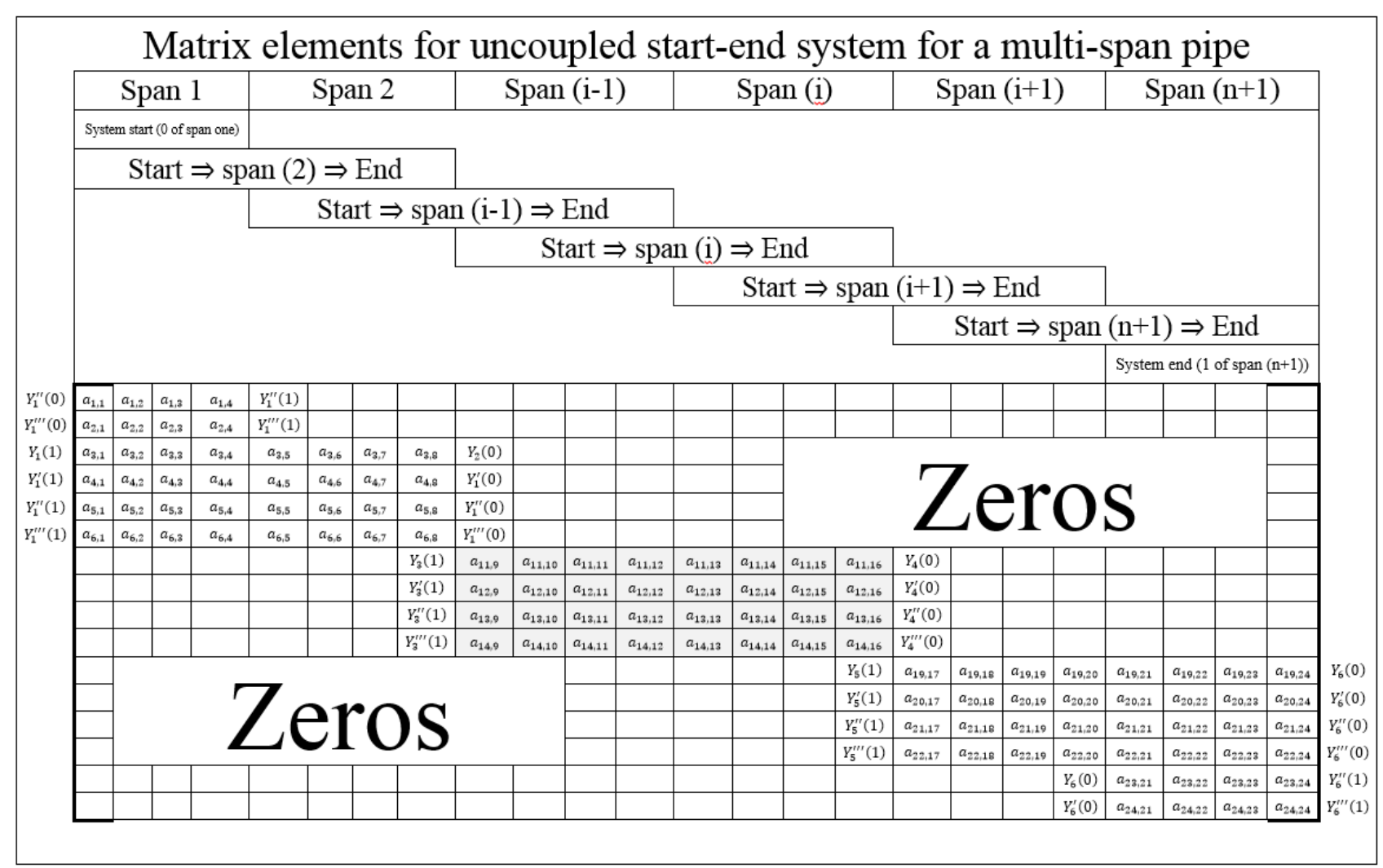

Fig. 2. Suggested matrix trend for elements of a multi-span pipe conveying fluid.

The elements of the matrix can be written as follows:

Start elements of the system, with span (1):

$$
\begin{array}{ll}
a_{11}=\kappa_{11}^{2}-\Phi_{1} \kappa_{11} \quad a_{12}=\kappa_{21}^{2}-\Phi_{1} \kappa_{21} & a_{13}=\kappa_{31}^{2}-\Phi_{1} \kappa_{31} \quad a_{14}=\kappa_{41}^{2}-\Phi_{1} \kappa_{41} \\
a_{21}=\kappa_{11}^{3}-\left(m_{1}^{*} \lambda_{1}^{4}+Z_{1}\right) & a_{22}=\kappa_{21}^{3}-\left(m_{1}^{*} \lambda_{1}^{4}+Z_{1}\right) \\
a_{23}=\kappa_{31}^{3}-\left(m_{1}^{*} \lambda_{1}^{4}+Z_{1}\right) & a_{24}=\kappa_{41}^{3}-\left(m_{1}^{*} \lambda_{1}^{4}+Z_{1}\right)
\end{array}
$$

Generalized intermediate elements of the system, start with span (2) to span (n+1):-

Where $S N$ is the span number $\Rightarrow i=n n-1, n n$ is the node number

\begin{tabular}{|l|l|l|l|l|l|l|l|}
\hline$a_{((4 i-1),(4 i-3))}$ & $a_{((4 i-1),(4 i-2))}$ & $a_{((4 i-1),(4 i-1))}$ & $a_{((4 i-1),(4 i))}$ & $a_{((4 i-1),(4 i+1))}$ & $a_{((4 i-1),(4 i+2))}$ & $a_{((4 i-1),(4 i+3))}$ & $a_{((4 i-1),(4 i+4))}$ \\
\hline$a_{((4 i),(4 i-3))}$ & $a_{((4 i),(4 i-2))}$ & $a_{((4 i),(4 i-1))}$ & $a_{((4 i),(4 i))}$ & $a_{((4 i),(4 i+1))}$ & $a_{((4 i),(4 i+2))}$ & $a_{((4 i),(4 i+3))}$ & $a_{((4 i),(4 i+4))}$ \\
\hline$a_{((4 i+1),(4 i-3))}$ & $a_{((4 i+1),(4 i-2))}$ & $a_{((4 i+1),(4 i-1))}$ & $a_{((4 i+1),(4 i))}$ & $a_{((4 i+1),(4 i+1))}$ & $a_{((4 i+1),(4 i+2))}$ & $a_{((4 i+1),(4 i+3))}$ & $a_{((4 i+1),(4 i+4))}$ \\
\hline$a_{((4 i+2),(4 i-3))}$ & $a_{((4 i+2),(4 i-2))}$ & $a_{((4 i+2),(4 i-1))}$ & $a_{((4 i+2),(4 i))}$ & $a_{((4 i+2),(4 i+1))}$ & $a_{((4 i+2),(4 i+2))}$ & $a_{((4 i+2),(4 i+3))}$ & $a_{((4 i+2),(4 i+4))}$ \\
\hline$a_{((4 i-1),(4 i-3))}=e^{\kappa_{1 i}}$, & $a_{((4 i-1),(4 i-2))}=e^{\kappa_{2 i}}$, & $a_{((4 i-1),(4 i-1))}=e^{\kappa_{3 i}}$, & $a_{((4 i-1),(4 i))}=e^{\kappa_{4 i}}$ \\
$a_{((4 i-1),(4 i+1))}=-1$, & $a_{((4 i-1),(4 i+2))}=-1$, & $a_{((4 i-1),(4 i+3))}=-1$, & $a_{((4 i-1),(4 i+4))}=-1$ \\
$a_{((4 i),(4 i-3))}=\kappa_{1 i} e^{\kappa_{1 i}}$ & $a_{((4 i),(4 i-2))}=\kappa_{2 i} e^{\kappa_{2 i}}$ & $a_{((4 i),(4 i-1))}=\kappa_{3 i} e^{\kappa_{3 i}}$ & $a_{((4 i),(4 i))}=\kappa_{4 i} e^{\kappa_{4 i}}$ \\
$a_{((4 i),(4 i+1))}=-L_{i} / L_{(i+1)} \kappa_{1(i+1),}$, & $a_{((4 i),(4 i+2))}=-L_{i} / L_{(i+1)} \kappa_{2(i+1)}$ \\
$a_{((4 i),(4 i+3))}=-L_{i} / L_{(i+1)} \kappa_{3(i+1)}$, & $a_{((4 i),(4 i+4))}=-L_{i} / L_{(i+1)} \kappa_{4(i+1)}$
\end{tabular}




$$
\begin{aligned}
& \text { put, } \quad W_{1 i}=E_{(i+1)} I_{(i+1)} L_{i}^{2} / E_{i} I_{i} L_{(i+1)}^{2} \\
& a_{((4 i+1),(4 i+1))}=-\left(W_{1 i} \kappa_{1(i+1)}^{2}-\left(L_{i} / L_{(i+1)}\right) \Phi_{i(i+1)} \kappa_{1(i+1)}\right) \text {, } \\
& a_{((4 i+1),(4 i+2))}=-\left(W_{1 i} \kappa_{2(i+1)}^{2}-\left(L_{i} / L_{(i+1)}\right) \Phi_{i(i+1)} \kappa_{2(i+1)}\right), \\
& a_{((4 i+1),(4 i+3))}=-\left(W_{1 i} \kappa_{3(i+1)}^{2}-\left(L_{i} / L_{(i+1)}\right) \Phi_{i(i+1)} \kappa_{3(i+1)}\right), \\
& a_{((4 i+1),(4 i+4))}=-\left(W_{1 i} \kappa_{4(i+1)}^{2}-\left(L_{i} / L_{(i+1)}\right) \Phi_{i(i+1)} \kappa_{4(i+1)}\right) \\
& a_{((4 i+1),(4 i-3))}=\kappa_{1 i}^{2} e^{\kappa_{1 i}}, \quad a_{((4 i+1),(4 i-2))}=\kappa_{2 i}^{2} e^{\kappa_{2 i}} \\
& a_{((4 i+1),(4 i-1))}=\kappa_{3 i}^{2} e^{\kappa_{3 i}}, \quad a_{((4 i+1),(4 i))}=\kappa_{4 i}^{2} e^{\kappa_{4 i}} \\
& a_{((4 i+2),(4 i+1))}=-\left(W_{2 i} \kappa_{1(i+1)}^{3}+\left(-m_{(i+1)}^{*} \times \lambda_{i}^{4}+Z_{(i+1)}\right)\right) \text {, } \\
& a_{((4 i+2),(4 i+2))}=-\left(W_{2 i} \kappa_{2(i+1)}^{3}+\left(-m_{(i+1)}^{*} \times \lambda_{i}^{4}+Z_{(i+1)}\right)\right) \text {, } \\
& a_{((4 i+2),(4 i+3))}=-\left(W_{2 i} \kappa_{3(i+1)}^{3}+\left(-m_{(i+1)}^{*} \times \lambda_{i}^{4}+Z_{(i+1)}\right)\right) \text {, } \\
& a_{((4 i+2),(4 i+4))}=-\left(W_{2 i} \kappa_{4(i+1)}^{3}+\left(-m_{(i+1)}^{*} \times \lambda_{i}^{4}+Z_{(i+1)}\right)\right) \\
& a_{((4 i+2),(4 i-3))}=\kappa_{1 i}^{3} e^{\kappa_{1 i}} \text {, } \\
& a_{((4 i+2),(4 i-2))}=\kappa_{2 i}^{3} e^{\kappa_{2 i}}, \\
& a_{((4 i+2),(4 i-1))}=\kappa_{3 i}^{3} e^{\kappa_{3 i}}, \quad a_{((4 i+2),(4 i))}=\kappa_{4 i}^{3} e^{\kappa_{4 i}} \\
& \text { put, } \quad W_{2 i}=E_{(i+1)} I_{(i+1)} L_{i}^{3} / E_{i} I_{i} L_{(i+1)}^{3}
\end{aligned}
$$

End elements of the system, with for span $(n+1)$ or (6):

$$
\begin{array}{ll}
a_{(23,21)}=\kappa_{16}^{2}+\Phi_{7} \kappa_{16} e^{\kappa_{16}} & a_{(23,22)}=\kappa_{26}^{2}+\Phi_{7} \kappa_{26} e^{\kappa_{26}} \\
a_{(23,23)}=\kappa_{36}^{2}+\Phi_{7} \kappa_{36} e^{\kappa_{36}} & a_{(23,24)}=\kappa_{46}^{2}+\Phi_{7} \kappa_{46} e^{\kappa_{46}} \\
a_{(24,21)}=\left(\kappa_{16}^{3}+\left(m_{7}^{*} \times \lambda_{6}^{4}+Z_{7}\right)\right) e^{\kappa_{16}} & a_{(24,22)}=\left(\kappa_{26}^{3}+\left(m_{7}^{*} \times \lambda_{6}^{4}+Z_{7}\right)\right) e^{\kappa_{26}} \\
a_{(24,23)}=\left(\kappa_{36}^{3}+\left(m_{7}^{*} \times \lambda_{6}^{4}+Z_{7}\right)\right) e^{\kappa_{36}} & a_{(24,24)}=\left(\kappa_{46}^{3}+\left(m_{7}^{*} \times \lambda_{6}^{4}+Z_{7}\right)\right) e^{\kappa_{46}}
\end{array}
$$

The previous elements are the elements of the determinant which represents, the characteristic equation. This equation is solved to get the natural frequencies and associated mode shapes of the system using Matlab package and the bisection numerical method.

\section{Model verification and case study}

A single span clamped-pinned pipe is analyzed and verified without concentrated masses as shown in Table 1, with reference [15]. A pinned-pinned pipe is verified with reference [16] to check the accuracy of present model results. Good agreement is obtained as illustrated in table 2 . The present model with multi-span pipe is verified its results with reference [12]. The model consists of a clampedpinned pipe, carrying five equal concentrated masses. 
Table 1.The first two modes for a (c-p) pipe conveying fluid, $\beta=0.3$ and without concentrated mass.

\begin{tabular}{lcccccc}
\hline \multirow{2}{*}{$\bar{v}_{f}$} & \multicolumn{3}{c}{$\lambda_{i}^{2}$} & \multicolumn{3}{c}{$\lambda_{i}^{2}$} \\
\cline { 2 - 7 } & {$[15]$} & present & $\%$ & {$[15]$} & present & $\%$ \\
\hline 0 & 15.418 & 15.418 & 0.000 & 49.964 & 49.965 & 0.002 \\
0.1 & 15.414 & 15.414 & 0.000 & 49.961 & 49.961 & 0.000 \\
0.2 & 15.401 & 15.403 & 0.015 & 49.952 & 49.948 & -0.009 \\
0.3 & 15.381 & 15.385 & 0.023 & 49.936 & 49.926 & -0.019 \\
0.4 & 15.352 & 15.358 & 0.041 & 49.915 & 49.896 & -0.038 \\
0.5 & 15.315 & 15.325 & 0.062 & 49.887 & 49.857 & -0.059 \\
1 & 15.003 & 15.040 & 0.245 & 49.653 & 49.534 & -0.241 \\
1.5 & 14.472 & 14.552 & 0.551 & 49.260 & 48.989 & -0.552 \\
2 & 13.702 & 13.838 & 0.986 & 48.702 & 48.217 & -1.006 \\
2.5 & 12.657 & 12.859 & 1.569 & 47.971 & 47.205 & -1.623 \\
3 & 11.275 & 11.541 & 2.305 & 47.056 & 47.937 & 1.838 \\
3.5 & 9.433 & 9.744 & 3.192 & 45.942 & 45.393 & -1.209 \\
4 & 6.806 & 7.099 & 4.127 & 44.606 & 42.542 & -4.852 \\
4.499 & 0.0 & 0.000 & 0.000 & 43.019 & 40.347 & -6.624 \\
\hline
\end{tabular}

The first two frequency parameters are shown in Table 1. There is a small percentage error between the present work and Ref. [15] .This percentage error appears due to the different methods used to get these frequencies. Chellapilla and Simha [15], use Fourier series and Galerkin's technique, but the present work uses the exact solution and the bisection method to find the roots.

Table 2. The first five natural frequencies of the seven-span (p-p-p-p-p-p-p) pipe.

\begin{tabular}{ccccccc}
\hline \multirow{2}{*}{$v_{f}=m / s$} & Method & \multicolumn{5}{c}{ Natural frequency (rad/sec) } \\
& & $\lambda_{1}$ & $\lambda_{2}$ & $\lambda_{3}$ & $\lambda_{4}$ & $\lambda_{5}$ \\
\hline$v_{f}=0$ & present & 29.8715 & 84.6195 & 167.5772 & 259.5011 & 274.1713 \\
& {$[16]$} & 29.8700 & 84.6200 & 167.5800 & 259.5000 & 274.1700 \\
& Error \% & 0.005 & 0.001 & -0.002 & 0 & 0.001 \\
$v_{f}=10$ & present & 29.8328 & 84.5667 & 167.5188 & 259.4383 & 274.1102 \\
& {$[16]$} & 29.8200 & 84.5700 & 167.5300 & 259.4400 & 274.1000 \\
& Error \% & 0.043 & -0.004 & -0.007 & -0.001 & 0.004 \\
$v_{f}=15$ & present & 29.7847 & 84.5007 & 167.4458 & 259.3574 & 274.0338 \\
& {$[16]$} & 29.7600 & 84.5100 & 167.4600 & 259.3400 & 274.0200 \\
& Error \% & 0.083 & -0.011 & -0.009 & 0.007 & 0.005
\end{tabular}

Although Deng [16] considered Coriolis term when calculating the natural frequencies for his case study, the obtained results indicate that, the present model with its assumption of neglecting Coriolis term, has good agreement with [16]. For more clear vision, Table (2) 
contains a very small percentage error. In the current model, the results are illustrated and compared with the results of Kang [12], Figure 4 and Table 3. Both results are almost identical. Non-dimensional velocity is $\bar{v}_{f}^{2}=\frac{m_{f} v_{f}^{2} L^{2}}{E I}$ and the natural frequency parameter is $\lambda_{i}^{* 2}=\sqrt{\left(\rho A L^{4} \omega^{2} / E I\right)}$.

Table 3. The first three natural frequencies vs nondimensional flow velocities for the (c-p) pipe with concentrated masses.

\begin{tabular}{|c|c|c|c|}
\hline $\bar{v}_{f}$ & $\lambda_{1}^{2}$ & $\lambda_{2}^{2}$ & $\lambda_{3}^{2}$ \\
\hline 0 & 8.7127 & 31.0369 & 60.5558 \\
\hline 0.5 & 8.6600 & 30.9687 & 60.4931 \\
\hline 1 & 8.4999 & 30.7632 & 60.3044 \\
\hline 1.5 & 8.2255 & 30.4176 & 59.9885 \\
\hline 2 & 7.8239 & 29.9269 & 59.5432 \\
\hline 2.5 & 7.2723 & 29.2840 & 58.9654 \\
\hline 3 & 6.5303 & 28.4785 & 58.2510 \\
\hline 3.5 & 5.5168 & 27.4963 & 57.3944 \\
\hline 4 & 4.0227 & 26.3180 & 56.3887 \\
\hline 4.1 & 3.6162 & 26.0567 & 56.1690 \\
\hline 4.3 & 2.5697 & 25.5063 & 55.7103 \\
\hline 4.45 & 1.2296 & 25.0682 & 55.3491 \\
\hline $\bar{v}_{f, r r}=4.49$ & 0.0000 & 24.9475 & 55.2502 \\
\hline 4.5 & - & 24.9171 & 55.2254 \\
\hline 5 & - & 23.2554 & 53.8934 \\
\hline 5.5 & - & 21.2772 & 52.3793 \\
\hline 6 & - & 18.8953 & 50.6658 \\
\hline 6.5 & - & 15.9611 & 48.7305 \\
\hline 7 & - & 12.1771 & 46.5447 \\
\hline 7.5 & - & 6.5993 & 44.0692 \\
\hline 7.6 & - & 4.8772 & 43.5349 \\
\hline 7.65 & - & 3.7618 & 43.2625 \\
\hline 7.71 & - & 1.6829 & 42.9308 \\
\hline $\bar{v}_{f c r}=7.73$ & - & 0.0000 & 42.8191 \\
\hline 8 & - & - & 41.2500 \\
\hline 8.5 & - & - & 38.0088 \\
\hline 9 & - & - & 34.2240 \\
\hline 9.5 & - & - & 29.6896 \\
\hline 10 & - & - & 23.9972 \\
\hline 10.5 & - & - & 16.0440 \\
\hline 10.6 & - & - & 13.8920 \\
\hline 10.7 & - & - & 11.3471 \\
\hline 10.8 & - & - & 8.0677 \\
\hline 10.9 & - & - & 1.5946 \\
\hline $\bar{v}_{f c r}=10.91$ & - & - & 0.0000 \\
\hline
\end{tabular}




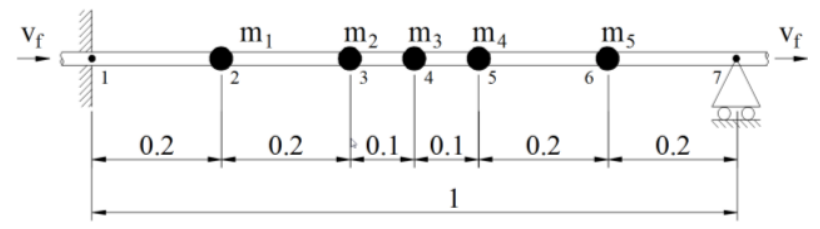

Fig. 3. Clamped-pinned pipe model [12] without intermediate support.

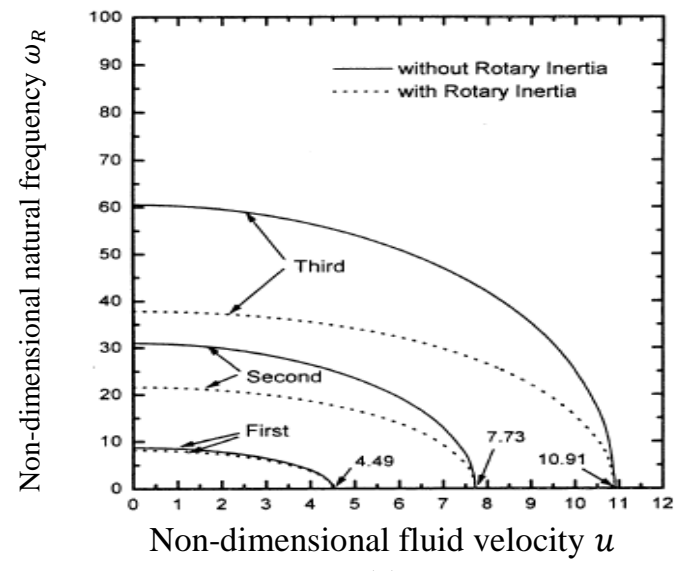

(a)

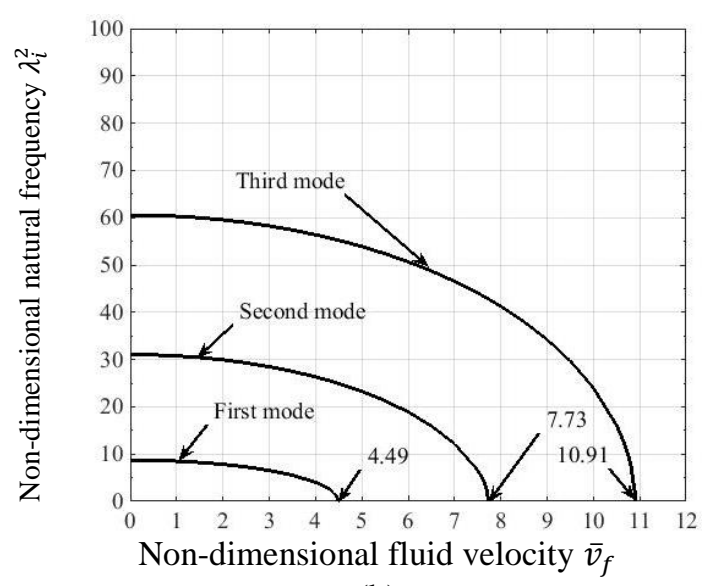

(b)

Fig. 4. The first three modes for a clamped-pinned pipe conveying fluid, (a) [12] and (b) present With $v_{f}=0.3, \beta=0.3, m_{1}^{*}=m_{2}^{*}=m_{3}^{*}=m_{4}^{*}=m_{5}^{*}=0.3$.

The case study is developed for clamped-pinned-pinned pipe as shown in Fig. 5 to study the effect of the intermediate support location on the critical flow velocity for different values of concentrated masses. Fig. 6 shows the natural frequencies vs the flow velocity for the first three modes with three locations for the intermediate support and four different values of the middle concentrated mass.

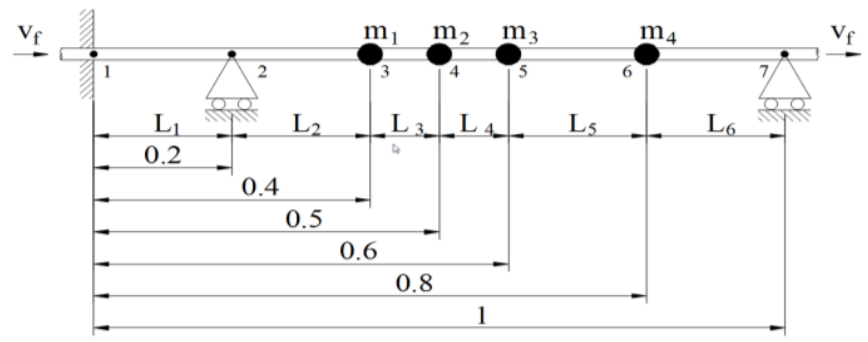

Fig. 5. Clamped-pinned-pinned pipe model with present intermediate support.

The values of the four concentrated masses $m_{i}^{*}$ are equal, where $(i=1,2,3,4)$ their non-dimensional values are: $0.1,0.2,0.3$ and 0.4 respectively. As can be seen from Fig. 6, the values of the natural frequencies are decreased due to the increase in concentrated mass values. At zero natural frequencies, the critical value of fluid flowing velocities are determined (for details see Table 3 and for the same location of the intermediate support, with the different values of the concentrated masses. The critical velocity is kept constant for each mode. 


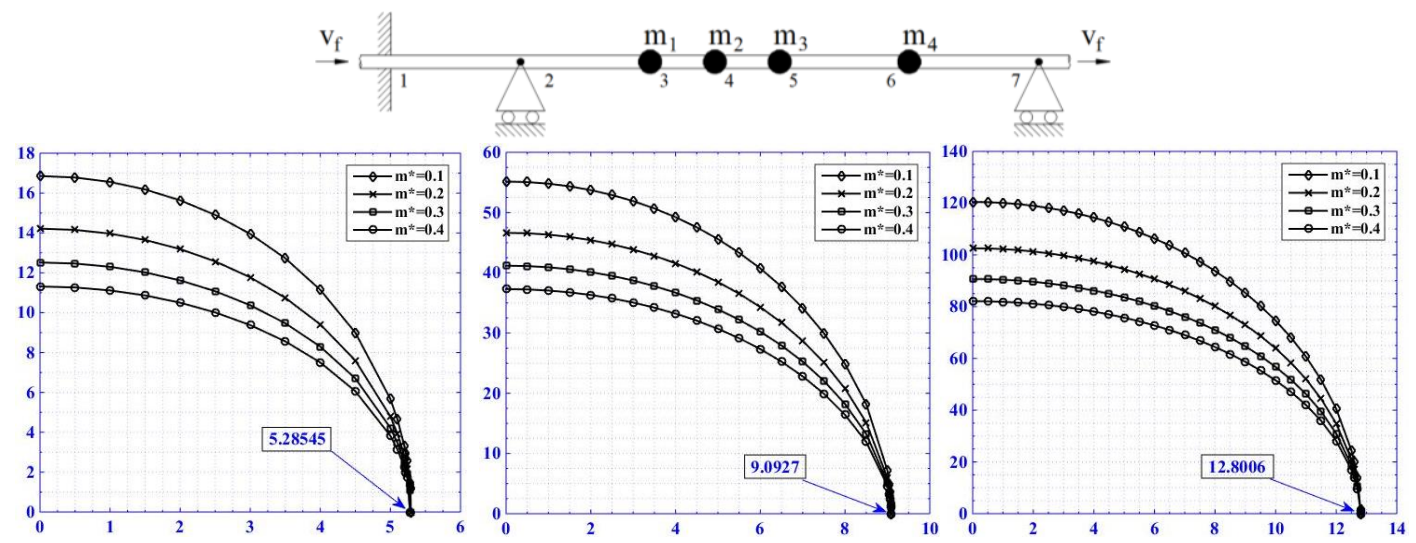

(a) first three modes, intermediate support at point (2).

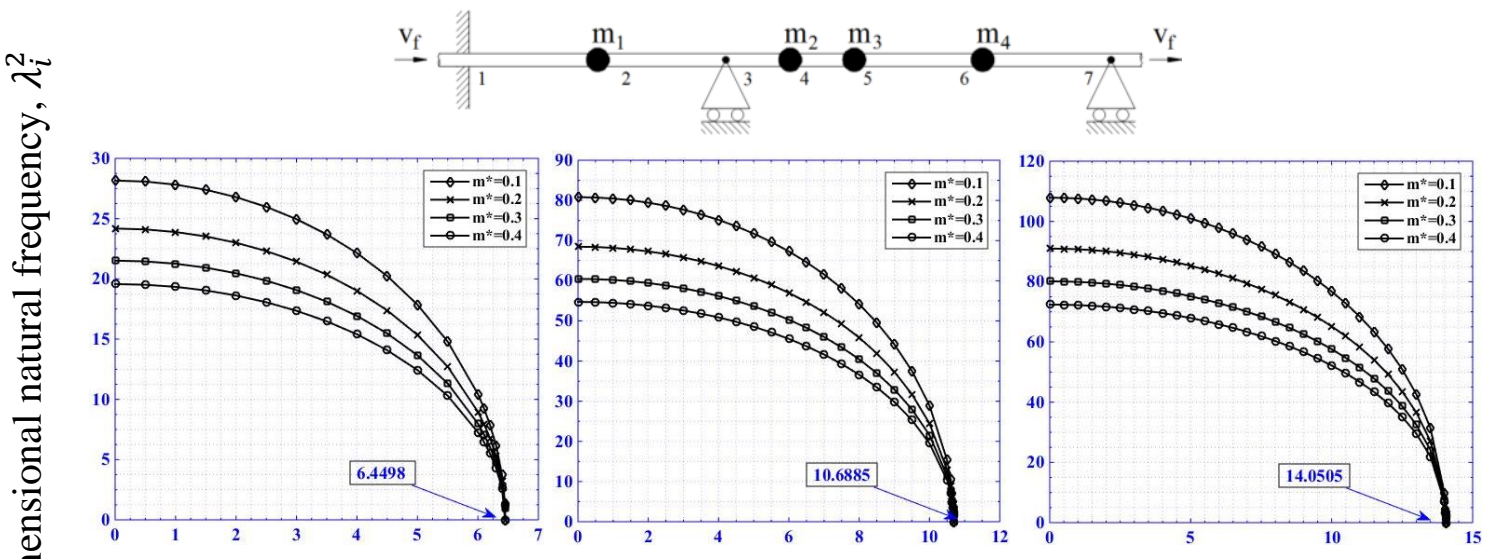

(b) first three modes, intermediate support at point (3).
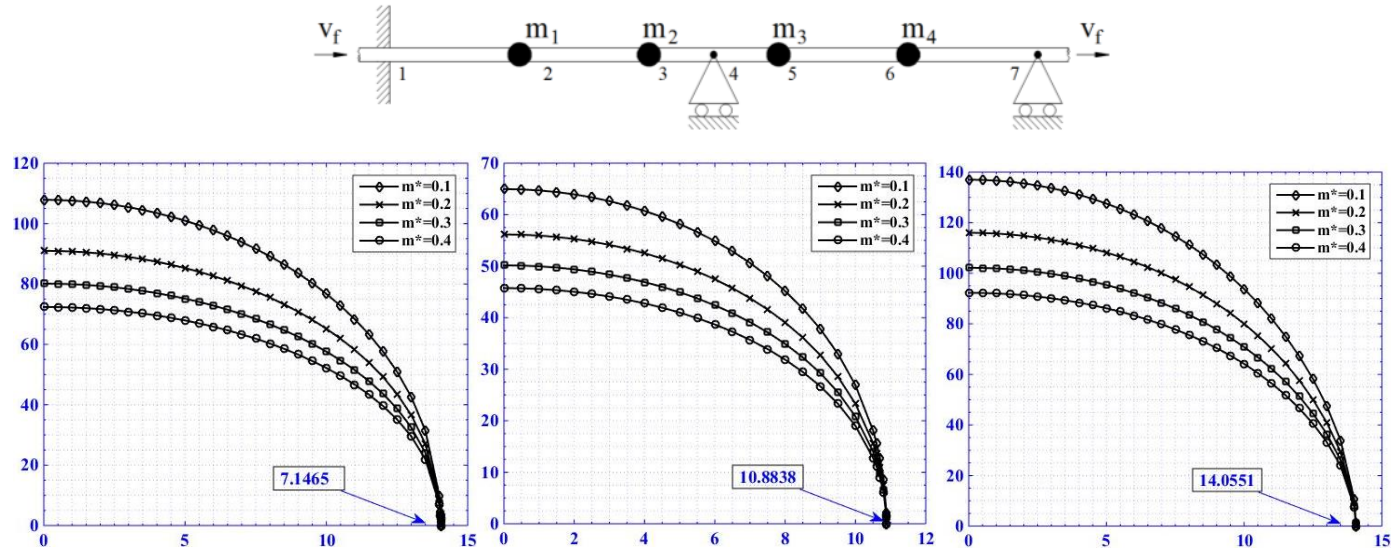

(c) first three modes, intermediate support at point (4).

non-dimensional fluid velocity, $\bar{v}_{f}$

Fig. 6. The first three modes vs flow velocity for different concentrated masses values and the intermediate support locations for a (c-p-p) pipe, (a) at point 2 ,

(b) at point 3 and (c) at point 4.

In Fig. 6, two parameters are studied. The first one is the concentrated mass parameter which varies, as $(0.1,0.2,0.3$ and 0.4$)$. The second one is the intermediate support location with three locations are selected for study. The three locations are represented by point (2,3 and 4$)$ which are assigned on the pipe model. As can be 
seen from Fig. 6, an increase in concentrated mass decreases the natural frequencies for the first three modes of vibration.

Intermediate support is located firstly at point (2). The first three natural frequencies are obtained. Comparing case one with case two at which the location is at point (3), the natural frequencies are increased. In the third case of location point (4), the natural frequencies are also increased. As can be seen from Fig. 6, the concentrated masses have no effect on the buckling critical velocities because buckling is a static phenomenon which depends on the elastic properties of the pipe rather than the inertial ones [9].
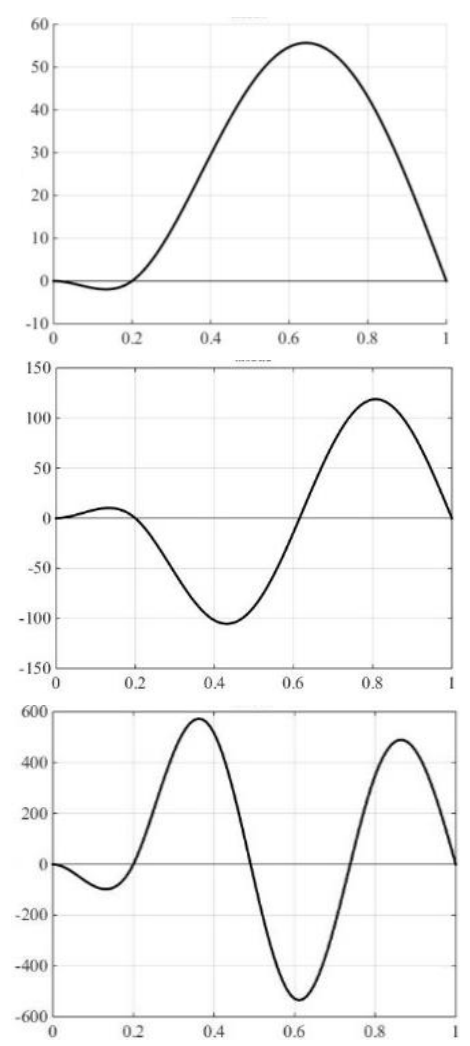

Support is at point 2.
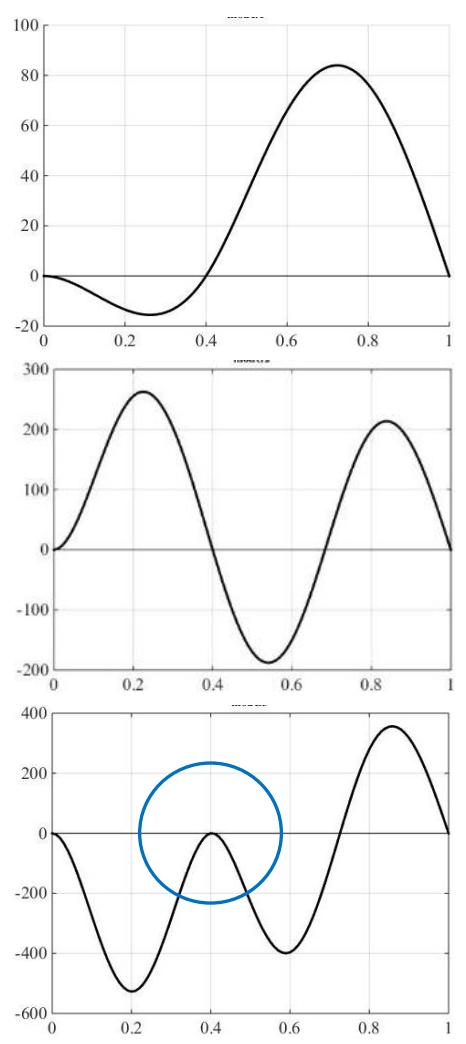

Support is at point 3.
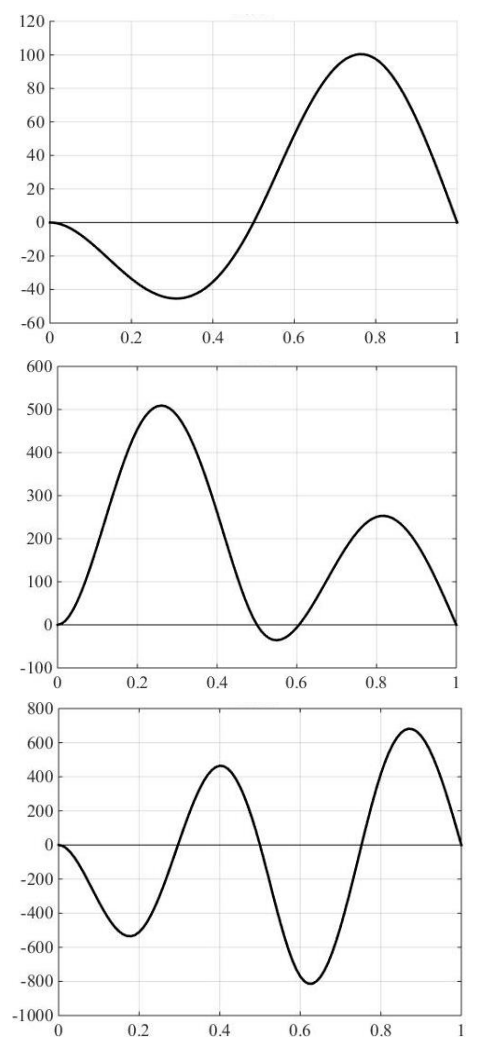

Support is at point 4 .

Fig. 7.The first three mode shapes of the (c-p-p) pipe with different locations of the intermediate support, $\left(m_{i}^{*}=0.3\right)$ and $\bar{v}_{f}=0.3$

In figure 7 , the case of intermediate support located at point 3 , two nodal points are lied around point 3 , see figure 8 , with magnified view to point 3 . 


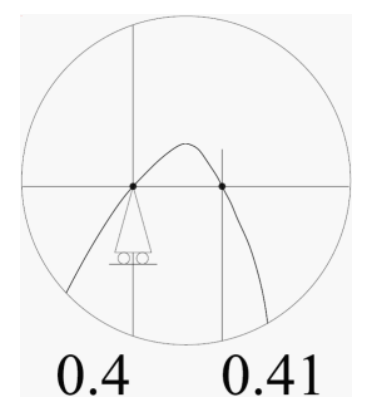

Fig. 8.Magnified area under mode shape curve, support is at point 3, shows

two nodal points at 0.4 and 0.41 .

\subsection{Mode shapes for the (c-p-p) pipe}

In pipe applications, the position of the nodal and anti-nodal points are of importance from the design point of view and for observability and controllability aspects. The control units have to be located at the nodes, while vibration measuring instrument are to be located at the anti-nodes. Fig. 7 shows the first three mode shapes of the (c-p-p) pipe with different locations of the intermediate support and the concentrated mass $\left(m_{i}^{*}=0.3\right)$. From the above figures, the shift of the nodes can be noticed due to the change of the intermediate support locations. The nodal points are important locations for any sensitive part to prevent their vibration.

\subsection{Effect of concentrated masses}

Table 3 shows the effect of concentrated masses on system natural frequencies at zero velocity of flow at different locations of the intermediate support. Referring to Table 4, an increase in the value of concentrated masses decreases the natural frequencies. For the first mode, an increase in the natural frequencies with the intermediate support location increases, from $0.2 L$ for $\lambda_{1, \min }^{2}$ to $0.5 L$, for $\lambda_{1, \max }^{2}$.

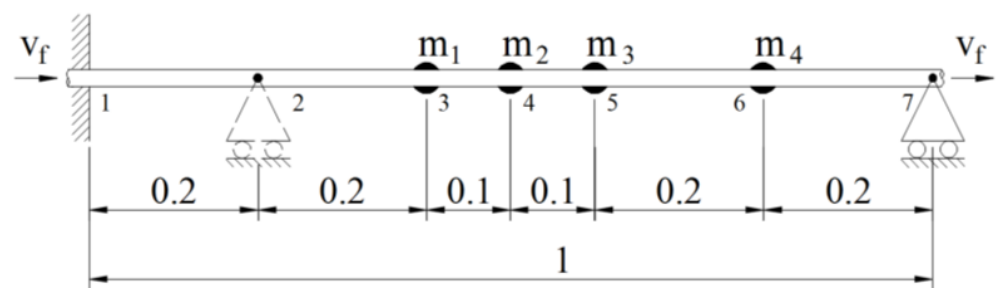

Fig. 8. Clamped-pinned pipe model with intermediate support. 
Table 4. The effect of concentrated masses and the intermediate support location on the natural frequencies for the first three modes for (c-p-p) pipe at $\bar{v}_{f}=0$.

\begin{tabular}{|c|c|c|c|c|c|c|}
\hline \multirow{2}{*}{$\begin{array}{c}\text { Intermediate } \\
\text { support } \\
\text { location } \\
\end{array}$} & & \multicolumn{5}{|c|}{ Concentrated masses } \\
\hline & & $m_{i}^{*}=0$ & $m_{i}^{*}=0.1$ & $m_{i}^{*}=0.2$ & $m_{i}^{*}=0.3$ & $m_{i}^{*}=0.4$ \\
\hline \multirow{3}{*}{ point (2) } & $\lambda_{1}^{2}$ & 21.8457 & 16.8537 & 14.2129 & 12.5180 & 11.3129 \\
\hline & $\lambda_{2}^{2}$ & 71.7316 & 55.1422 & 46.6319 & 41.1795 & 37.2927 \\
\hline & $\lambda_{3}^{2}$ & 150.8768 & 120.4664 & 102.5637 & 90.6861 & 82.1186 \\
\hline \multirow{3}{*}{ point (3) } & $\lambda_{1}^{2}$ & 35.0781 & 28.1589 & 24.1772 & 21.5126 & 19.5701 \\
\hline & $\lambda_{2}^{2}$ & 103.3874 & 80.8019 & 68.4705 & 60.4577 & 54.7228 \\
\hline & $\lambda_{3}^{2}$ & 139.2128 & 107.8757 & 90.9763 & 80.1090 & 72.3822 \\
\hline \multirow[t]{3}{*}{ point (4) } & $\lambda_{1}^{2}$ & 46.0561 & 37.5898 & 32.5379 & 29.0916 & 26.5490 \\
\hline & $\lambda_{2}^{2}$ & 79.6851 & 64.9674 & 56.1646 & 50.1661 & 45.7473 \\
\hline & $\lambda_{3}^{2}$ & 171.3698 & 137.0024 & 116.0178 & 102.1692 & 92.2552 \\
\hline
\end{tabular}

\subsection{Critical velocity}

Table 5 represents the critical velocities values, related to intermediate support locations and different values of concentrated masses. The values in this table are used to support the curves illustrated in Fig. 6.

Table 5. The effect of intermediate support location on the critical velocities for the first three modes for a (c-p-p) pipe.

\begin{tabular}{lccc}
\hline Vibration & \multicolumn{3}{c}{$\bar{V}_{c r}$} \\
\cline { 2 - 4 } \multicolumn{1}{c}{ mode } & $0.2 L$ & $0.4 L$ & $0.5 L$ \\
\hline First & 5.2855 & 6.4498 & 7.1465 \\
Second & 9.0927 & 10.6885 & 10.8838 \\
Third & 12.8006 & 14.0505 & 14.0551 \\
\hline
\end{tabular}

Referring to Table 5, an increase in the critical velocity is observed due to the change of the support location towards the middle of the pipe. Hence the maximum critical velocity of flow is observed at the middle point (4) of $0.5 \mathrm{~L}$ for all three modes.

\section{Conclusion}

The main conclusions from the present work can be withdrawn as follows;

- An analytical model and a computer code for multi-span pipe conveying fluid have been implemented using closed form solution.

- Good agreement between results of the present model and previous relevant publications are obtained. 
- The change of the nodal and anti-nodal points in the mode shape with the location of the intermediate support is observed.

- Interaction between the two parameters, the concentrated masses and the intermediate support locations affect the system vibration and its critical velocities.

\section{Nomenclature}

$A$ Cross-section of the pipe. $Y$ Lateral displacement of the pipe.

$E \quad$ Young's modulus. $\left(\mathrm{N} / \mathrm{m}^{2}\right) \quad Z_{1} \quad$ Lateral rigidity parameter

I Moment of inertia of the pipe section about the neutral axis. $\phi_{1}$ Bending stiffnesses.

$k_{1} \quad$ Translation spring stiffness. $\Phi_{1}$ Rotational rigidity parameter

$\kappa \quad$ The wave numbers.

$L$ Length of the

$m_{v}$ Mass of the pipe. $\lambda^{4}=\left(\rho A L^{4} \omega^{2} / E I\right)$

$m_{f} \quad$ Fluid mass. $\quad \omega$ Circular frequency.

$m_{i}^{*} \quad$ Concentrated mass parameter, $\rho_{p}$ Density of the pipe material.

$$
m_{i}^{*}=m_{i} / L\left(m_{f}+m_{n}\right) \quad \xi \quad \text { Non-dimensional pipe length, }
$$

$v_{f} \quad$ Steady flow velocity of fluid. $\quad C \quad$ Clamped (fixed) support.

$\bar{v}_{f} \quad$ Flow velocity parameter, $\quad P \quad$ Pinned (hinged) support.

$$
\bar{v}_{f}^{2}=m_{f} v_{f}^{2} L^{2} / E I \quad \text { vs } \quad \text { Very small, }(10 E-8) .
$$

$x, y \quad$ System co-ordinate of the pipe. $\quad v l$ Very large, $(10 E+8)$.

\section{References}

1. Ashley H., Havilland G. Bending vibration of pipe line containing flowing fluid, J. Appl. Mech., 17, 1950, pp. 229-232.

2. Housner, G.E. Bending vibration of a pipe line containing flowing fluid, Trans. ASME J. Appl. Mech., 74, 1952, pp. 205- 208.

3. Benjamin, T.B. Dynamics of a system of articulated pipes conveying fluid, I. Theory, Proceedings of the Royal Society of London. Series A, Math. Phys. Sci. (261) 1961a, pp. 457-486.

4. Benjamin, T.B. Dynamics of a system of articulated pipes conveying fluid, II. Experiments, Proceedings of the Royal Society of London. Series A, Math. Phys. Sci. (261) 1961b, pp. 487-499.

5. Dodds H. L., Jr., H. L. Runyan. Effect of high-velocity fluid flow on the bending vibrations and static divergence of a simply supported pipe, NASA TN D-2870, 1965, pp. 1-13. 
6. Blevins, R.D. Flow-induced vibration, Van Nostrand Reinhold, New York, 1977, pp. 287-312.

7. C. N. Bapat, C. Bapat. Natural frequencies of a beam with non-classical boundary conditions and concentrated masses, Journal of Sound and Vibration 112(1), 1987, pp. 177-182.

8. A. A. AL-Rajihy. Out-of-plane vibration and stability of intermediately supported curved-straight tube conveying fluid, M. Sc. Thesis, 1990.

9. S. H. Farghaly, E. M. Rabeih, M. M. El-Maddah. Fluid-structure interaction in a vibrating piping system, AME $5^{\text {th }}$ Conf., 1992, pp. 13-26.

10. S.H. Farghaly, T.A. El-Sayed. Exact free vibration analysis for mechanical system composed of Timoshenko beams with intermediate eccentric rigid body on elastic supports: An experimental and analytical investigation, Mechanical Systems and Signal Processing, 2016, pp. 1-18.

11. T.A. El-Sayed, S.H. Farghaly. Exact free vibration analysis of Timoshenko stepped shaft carrying elastically supported eccentric masses with application on SWRO mechanical system, Desalination, 2016, 385, pp.194-206.

12. Myeong-Gie Kang. The influence of rotary inertia of concentrated masses on the natural vibrations of a clamped-supported pipe conveying fluid, Nuclear Engineering and Design 196, 2000, pp. 281-292.

13. M. P. Païdoussis. Slender structures and axial flow fluid-structure interactions, Academic Press is an imprint of Elsevier, $2^{\text {nd }}$ edition, 2014, Vol. 1.

14. A. Kesimli, S. M. Bağdatli, S. Çanakci. Free Vibrations of Fluid Conveying Pipe by Intermediate Supported, Research on Engineering Structures \& Materials, 1, 2015, pp. 39-51.

15. K. R. Chellapilla, A and H.S. Simha. Vibrations of fluid-conveying pipes resting on two-parameter foundation, The Open Acoustics Journal, (1) 2008, pp. 24-33.

16. J. Deng, Y. Liu, Z. Zhang, W. Liu. Stability analysis of multi-span viscoelastic functionally graded material pipes conveying fluid using a hybrid method, European Journal of Mechanics / A Solids, Accepted Manuscript at April 2017. 